\title{
Intraplatelet Vascular Endothelial Growth Factor and Platelet-Derived Growth Factor: New Biomarkers in Carcinoembryonic Antigen-Negative Colorectal Cancer?
}

\author{
Charbel Chater $^{a} \quad$ Anne Bauters ${ }^{b} \quad$ Claire Beugnet $^{b}$ Lena $\mathrm{M}^{\prime} \mathrm{Ba}^{\mathrm{a}}$ \\ Moshe Rogosnitzky ${ }^{c}$ Philippe Zerbib ${ }^{a}$ \\ ${ }^{a}$ Digestive Surgery and Transplantation Unit, Hôpital Huriez, Lille University Medical Center, \\ Lille Nord de France University, Lille, France; ${ }^{b}$ Department of Hematology and Transfusion, \\ Centre de Biologie et Pathologie, Lille University Medical Center, Lille, France; ${ }^{\mathrm{C}}$ MedInsight \\ Research Institute, Telz-Stone, Israel
}

\author{
Keywords \\ Carcinoembryonic antigen - Colorectal cancer - Intraplatelet angiogenesis regulators . \\ Platelet-derived growth factor · Vascular endothelial growth factor
}

\begin{abstract}
Background/Aim: Colorectal cancer (CRC) is associated with high incidence and mortality rates. Carcinoembryonic antigen (CEA), a prognostic biomarker for recurrent CRC following curative resection, suffers from low sensitivity, especially in early-stage screening. Intraplatelet angiogenesis regulators (IPAR), such as vascular endothelial growth factor (VEGF) and platelet-derived growth factor (PDGF), have been identified as important regulators of tumor growth in CRC. The aim of this study was to confirm the higher preoperative level of IPAR (VEGF and PDGF) in CRC patients compared to controls and to measure IPAR in CEA-negative CRC patients. Methods: The data and blood of 30 CRC patients and 30 presumably healthy controls were prospectively analyzed and compared. Results: We confirmed elevated preoperative intraplatelet VEGF and PDGF levels in CRC patients compared to controls. Importantly, IPAR were significantly elevated even in CEA-negative CRC patients. Conclusion: Elevated preoperative intraplatelet VEGF and PDGF levels in CRC patients suggest new possibilities for postoperative monitoring in CRC patients, especially when CEA is negative.
\end{abstract}

(C) 2018 S. Karger AG, Basel 


\section{Introduction}

Colorectal cancer (CRC) is the third most common cancer diagnosed and results in high mortality rates [1]. Hepatic metastasis is the leading cause of systemic recurrence and death in CRC [2], making its prevention a major challenge [3, 4]. Serum carcinoembryonic antigen (CEA) testing is recommended by the American Society of Clinical Oncology [5] and the European Group on Tumor Markers [6] as a prognostic biomarker for recurrent CRC following curative resection. CEA suffers from low sensitivity since most patients have negative preoperative CEA levels [7]. These patients lack a postoperative biologic prognostic factor for identifying tumor recurrence. There is a need to identify new biomarkers to enhance early cancer detection, improve patient stratification, and predict therapeutic response.

Trousseau's work on plasmatic hypercoagulability associated with cancer has revealed the implication of platelets in different phases of cancer progression [8, 9]. Indeed, its implication has been clinically confirmed by the efficacy of aspirin in the prevention of CRC in humans [10]. Very early in the course of cancer development, angiogenesis regulators are taken up, internalized, and concentrated in platelet alpha granules [11] for delivery to tumors through specific activation [12]. These intraplatelet angiogenesis regulators (IPAR) have been implicated in tumor growth and invasion [13]. Among them, vascular endothelial growth factor (VEGF) and platelet-derived growth factor (PDGF) play an important role in CRC development [14].

The aim of this study was to confirm higher preoperative levels of IPAR (VEGF and PDGF) in CRC patients compared to controls, and then to measure IPAR in CEA-negative CRC patients.

\section{Methods}

\section{Study Population}

We prospectively analyzed the data of 30 CRC patients and compared them to those of 30 presumably healthy controls. CRC patients were treated in the Regional Hospital of Lille. We included all consecutive CRC patients undergoing curative treatment. Patients presenting sepsis (or infection), liver cirrhosis, chronic kidney disease, diabetes, vasculitis, extrahepatic metastasis, or history of inflammatory disease, or patients needing urgent surgery were excluded. Healthy individuals were the first 30 volunteers among the hospital staff with no history of CRC, inflammatory bowel disease, or other disease. Healthy individuals received no treatment.

Blood Samples

For CRC patients, peripheral venous blood samples were collected in EDTA prior to surgery and centrifuged. Plasma and platelet pellets were stored at $-80^{\circ} \mathrm{C}$ until analysis in accordance with Peterson et al. [15].

Analysis

Intraplatelet VEGF and PDGF were measured using quantitative sandwich immunoassays (R\&D Systems, UK). Plasma P-selectin, a platelet biomarker, was also analyzed. The optical density levels of samples were converted to concentrations as predicted from calibration curves run on each microplate. Intraplatelet VEGF and PDGF levels were then adjusted to the number of platelets through expressing intraplatelet biomarker concentrations per actin unit, as actin is a surrogate marker of platelet size and count (PathScan Total $\beta$-Actin Sandwich ELISA Kit; Ozyme, France). Serum CEA values up to $10 \mathrm{ng} / \mathrm{mL}$ were considered negative.

Statistical Analysis

Variables with skewed distribution were log-transformed before use as continuous variables in statistical analyses. Continuous variables were compared with unpaired Student $t$ test. Discrete variables were compared using $\chi^{2}$ analysis. A $p$ value $<0.05$ was considered statistically significant (two-sided). Multivariate analysis adjusted for age was used to determine the independent predictors of CRC. Statistical analysis was conducted using the SPSS software (SPSS Inc./IBM, Chicago, IL, USA). 
Table 1. Characteristics of the patients and controls

\begin{tabular}{lccc}
\hline & $\begin{array}{l}\text { CRC patients } \\
(n=30)\end{array}$ & $\begin{array}{l}\text { Controls } \\
(n=30)\end{array}$ & $p$ value \\
\hline $\begin{array}{l}\text { Age, years } \\
\text { Sex }\end{array} \quad 63(12.6)$ & $47(11.3)$ & $<0.001$ \\
$\quad$ Male & 19 & 16 & \\
$\quad$ Female & 11 & 14 & \\
$\quad$ Male $/$ female & & & \\
Platelets, $\times 10^{9} / \mathrm{L}$ & $259(148)$ & $246(39)$ & 0.6 \\
Tumor location & & & \\
$\quad$ Colon & 22 & & \\
$\quad$ Rectum & 8 & & \\
TNM stage & & \\
$\quad$ Stage I & 6 & \\
$\quad$ Stage II & 8 & \\
$\quad$ Stage III & 8 & \\
$\quad$ Stage IV & 8 & \\
CEA & & \\
$\quad<10$ ng/mL & 24 & \\
$\quad>10 \mathrm{ng} / \mathrm{mL}$ & 6 & \\
\hline
\end{tabular}

Values are presented as number of subjects or mean (standard deviation). CEA, carcinoembryonic antigen.

Table 2. Biomarkers in healthy controls, CRC patients, and the CEA-negative CRC subset

\begin{tabular}{|c|c|c|c|c|c|}
\hline Variables & $\begin{array}{l}\text { Controls } \\
(n=30)\end{array}$ & $\begin{array}{l}\text { CRC patients } \\
(n=30)\end{array}$ & $p$ value & $\begin{array}{l}\text { CEA-negative CRC } \\
\text { subset }(n=24)\end{array}$ & $p$ value \\
\hline VEGF, pg/actin unit & $2.59(0.22-22.37)$ & $15.55(1.38-693.05)$ & $<0.001$ & $13.97(2.16-693.05)$ & $<0.001$ \\
\hline PDGF, pg/actin unit & $125(33-641)$ & $304(49-8,807)$ & 0.001 & $304(49-8,807)$ & 0.001 \\
\hline Soluble P-selectin, ng/mL & $60.2(36.40-82.70)$ & $69.25(33.80-152)$ & 0.004 & $65.8(33.80-152)$ & 0.01 \\
\hline
\end{tabular}

Values are presented as median (range). CEA, carcinoembryonic antigen; CRC, colorectal cancer; PDGF, platelet-derived growth factor; VEGF, vascular endothelial growth factor.

\section{Results}

The characteristics of the patients and controls are shown in Table 1 . The healthy patients were younger than the CRC patients. Among the CRC patients, tumor location was mainly in the colon.

\section{Higher Platelet Activation in CRC Patients}

The median values of plasma P-selectin was higher in the CRC patients $(69.25 \mathrm{ng} / \mathrm{mL})$ than in the controls $(60.2 \mathrm{ng} / \mathrm{mL})(p=0.004)$ (Table 2$)$.

\section{Higher IPAR in CRC Patients}

The median values of intraplatelet VEGF were higher in the CRC patients $(15.55 \mathrm{pg} /$ actin unit) than in the controls $(2.59 \mathrm{pg} /$ actin unit) $(p<0.001)$ (Table 2). Similarly, the median values of intraplatelet PDGF were higher in the CRC patients (304 pg/actin unit) than in the controls (125 pg/actin unit) ( $p=0.001)$ (Table 2). 
Higher Platelet Activation and IPAR in CEA-Negative CRC Patients

In the subset of CEA-negative CRC patients $(n=24)$, the median values of plasma P-selectin and intraplatelet VEGF and PDGF were still significantly higher than in the controls (Table 2).

\section{VEGF and PDGF: Biomarkers Predicting CRC Independent of Age}

The healthy patients were significantly younger ( $47 \pm 11.3$ years) than the CRC patients $(63 \pm 12.6$ years) $(p<0.001)$ (Table 1$)$. In order to assess the effect of age on the biomarkers, we did a multivariate analysis adjusted for age; plasma P-selectin $(p=0.031)$, intraplatelet VEGF ( $p<0.001)$, and PDGF ( $p=0.002)$ levels were predictors of CRC independent of age.

\section{Discussion}

We confirmed elevated preoperative intraplatelet VEGF and PDGF in CRC patients compared to controls [13]. Importantly, we found that IPAR were significantly elevated even in CEA-negative patients.

Despite being the most widely accepted marker for CRC postsurgical recurrence, CEA has several drawbacks, in addition to not being detected at early stages of CRC [7]: CEA is negative in non-CEA-producing tumors [16]; moreover, measuring postoperative CEA levels in patients with negative preoperative values is meaningless because CEA remains negative during CRC recurrence [7, 17]. Finally, the addition of CEA testing to scheduled CT scanning did not improve detection of recurrence [18]. In our study, 80\% of CRC patients presented with negative CEA, which is in line with figures reported by others [7]. We did not find significant differences for platelet counts between patients and controls. In the natural course of cancer, there is a tendency for overconsumption of platelets during the formation of microemboli and overproduction through the paraneoplastic overexpression of thrombopoietin $[19,20]$. A normal platelet count reflects efficient compensatory mechanisms through production of immature and enzymatically more active platelets which facilitates activation in contact with tumor cells and release of proangiogenic proteins $[12,21]$. Higher IPAR load in platelets from CRC patients could be explained through enhanced production in bone marrow platelet progenitors as well as endocytosis of circulating angiogenesis regulators by platelets and/or megakaryocytes [13]. Measurement of IPAR represents several advantages over serum measurements of VEGF and PDGF: platelets do not completely release angiogenesis regulators in the serum [12], and the increase of IPAR seems to occur earlier and to be more specific than elevations of VEGF and PDGF in serum [11].

Some recent studies have suggested that biomarkers could be modified by environmental factors such as the type of food [22]. However, to the best of our knowledge, there is no study assessing the effect of environmental factors on VEGF and PDGF. Consequently, these factors were not assessed in our work.

Our pilot study is the first prospective study showing that preoperative assessment of intraplatelet VEGF and PDGF in CRC patients offers new possibilities for postoperative monitoring, especially when CEA is negative.

The next step would be to do a larger prospective study assessing these biomarkers in CRC patients compared to age-matched controls. Moreover, a colonoscopy should be performed for the control group in order to confirm the absence of any unknown mucosal lesion. 


\section{Conclusion}

Our finding of elevated preoperative intraplatelet VEGF and PDGF levels in CEA-negative CRC patients suggests new possibilities for postoperative monitoring in CRC patients, especially when CEA is negative.

\section{Acknowledgments}

The authors thank Carol A. Bienstock for assistance with manuscript preparation.

\section{Statement of Ethics}

Blood samples were collected after informed consent in accordance with the Declaration of Helsinki and institutional guidelines (No. CPP A00264-5).

\section{Disclosure Statement}

The authors report no conflicts of interest.

\section{References}

1 Siegel R, Naishadham D, Jemal A: Cancer statistics, 2013. CA Cancer J Clin 2013;63:11-30.

$\longrightarrow 2$ De Ridder JA, Lemmens VE, Overbeek LI, Nagtegaal ID, De Wilt JH; Dutch Liver Surgery Group: Liver resection for metastatic disease; a population-based analysis of trends. Dig Surg 2016;33:104-113.

-3 Pawlik TM, Schulick RD, Choti MA: Expanding criteria for resectability of colorectal liver metastases. Oncologist 2008;13:51-64.

4 Maeda C, Hidaka E, Mori Y, Mukai S, Miyachi H, Sawada N, Ishida F, Kudo SE: Tumor diameter is an easy and useful predictor of recurrence in stage II colorectal cancer. Dig Surg 2015;32:338-343.

5 Locker GY, Hamilton S, Harris J, Jessup JM, Kemeny N, Macdonald JS, Somerfield MR, Hayes DF, Bast RC Jr: ASCO 2006 update of recommendations for the use of tumor markers in gastrointestinal cancer. J Clin Oncol 2006; 24:5313-5327.

6 Duffy MJ, Van Dalen A, Haglund C, Hansson L, Holinski-Feder E, Klapdor R, Lamerz R, Peltomaki P, Sturgeon C, Topolcan O: Tumour markers in colorectal cancer: European Group on Tumour Markers (EGTM) guidelines for clinical use. Eur J Cancer 2007;43:1348-1360.

7 Park IJ, Choi GS, Lim KH, Kang BM, Jun SH: Serum carcinoembryonic antigen monitoring after curative resection for colorectal cancer: clinical significance of the preoperative level. Ann Surg Oncol 2009;16:3087-3093.

-8 Gay LJ, Felding-Habermann B: Contribution of platelets to tumour metastasis. Nat Rev Cancer 2011;11:123134.

9 Erpenbeck L, Schon MP: Deadly allies: the fatal interplay between platelets and metastasizing cancer cells. Blood 2010;115:3427-3436.

10 Rothwell PM, Fowkes FG, Belch JF, Ogawa H, Warlow CP, Meade TW: Effect of daily aspirin on long-term risk of death due to cancer: analysis of individual patient data from randomised trials. Lancet 2011;377:31-41.

$\checkmark 11$ Klement GL, Yip TT, Cassiola F, Kikuchi L, Cervi D, Podust V, Italiano JE, Wheatley E, Abou-Slaybi A, Bender E, Almog N, Kieran MW, Folkman J: Platelets actively sequester angiogenesis regulators. Blood 2009;113:28352842.

12 Italiano JE Jr, Richardson JL, Patel-Hett S, Battinelli E, Zaslavsky A, Short S, Ryeom S, Folkman J, Klement G: Angiogenesis is regulated by a novel mechanism: pro- and antiangiogenic proteins are organized into separate platelet alpha granules and differentially released. Blood 2008;111:1227-1233.

13 Peterson JE, Zurakowski D, Italiano JE Jr, Michel LV, Connors S, Oenick M, D’Amato RJ, Klement GL, Folkman J: VEGF, PF4 and PDGF are elevated in platelets of colorectal cancer patients. Angiogenesis 2012;15:265-273.

14 Folkman J: Angiogenesis: an organizing principle for drug discovery? Nat Rev Drug Discov 2007;6:273-286.

15 Peterson JE, Zurakowski D, Italiano JE Jr, Michel LV, Fox L, Klement GL, Folkman J: Normal ranges of angiogenesis regulatory proteins in human platelets. Am J Hematol 2010;85:487-493.

16 Su BB, Shi H, Wan J: Role of serum carcinoembryonic antigen in the detection of colorectal cancer before and after surgical resection. World J Gastroenterol 2012;18:2121-2126. 
17 Yakabe T, Nakafusa Y, Sumi K, Miyoshi A, Kitajima Y, Sato S, Noshiro H, Miyazaki K: Clinical significance of CEA and CA19-9 in postoperative follow-up of colorectal cancer. Ann Surg Oncol 2010;17:2349-2356.

-18 Jones RP, McWhirter D, Fretwell VL, McAvoy A, Hardman JG: Clinical follow-up does not improve survival after resection of stage I-III colorectal cancer: a cohort study. Int J Surg 2015;17:67-71.

19 Dymicka-Piekarska V, Kemona H: Thrombopoietin and reticulated platelets as thrombopoietic markers in colorectal cancer. Thromb Res 2008;122:141-143.

20 Seretis C, Youssef H, Chapman M: Hypercoagulation in colorectal cancer: what can platelet indices tell us? Platelets 2015;26:114-118.

21 Sciulli MG, Filabozzi P, Tacconelli S, Padovano R, Ricciotti E, Capone ML, Grana M, Carnevale V, Patrignani P: Platelet activation in patients with colorectal cancer. Prostaglandins Leukot Essent Fatty Acids 2005;72: 79-83.

22 Kanellos PT, Kaliora AC, Protogerou AD, Tentolouris N, Perrea DN, Karathanos VT: The effect of raisins on biomarkers of endothelial function and oxidant damage; an open-label and randomized controlled intervention. Food Res Int 2017;102:674-680. 